\title{
Corela
}

Cognition, représentation, langage

HS-1 | 2005

Colloque AFLS

\section{L'acquisition de la liaison en français langue seconde}

Une analyse quantitative d'apprenants avancés en milieu guidé et en milieu naturel

\section{Martin Howard}

\section{OpenEdition}

Journals

Édition électronique

URL : http://journals.openedition.org/corela/1127

DOI : 10.4000/corela. 1127

ISSN : 1638-573X

Éditeur

Cercle linguistique du Centre et de l'Ouest - CerLICO

Référence électronique

Martin Howard, "L'acquisition de la liaison en français langue seconde », Corela [En ligne], HS-1 | 2005, mis en ligne le 16 février 2005, consulté le 30 avril 2019. URL : http://journals.openedition.org/ corela/1127; DOI : 10.4000/corela.1127

Ce document a été généré automatiquement le 30 avril 2019

\section{(c) (i) (3) (2)}

Corela - cognition, représentation, langage est mis à disposition selon les termes de la licence Creative Commons Attribution - Pas d'Utilisation Commerciale - Partage dans les Mêmes Conditions 4.0 International. 


\section{L'acquisition de la liaison en français langue seconde}

Une analyse quantitative d'apprenants avancés en milieu guidé et en milieu naturel ${ }^{1}$

\section{Martin Howard}

\section{Introduction}

1 L'étude que présente cet article fait partie du nouveau courant de recherche en acquisition des langues secondes qui vise à cerner l'appropriation de la variation sociolinguistique par le locuteur L2. Comme l'indiquent Mougeon et coll. (2003), la recherche traditionnelle sur la variation linguistique en L2 se ciblait principalement sur l'alternance chez l'apprenant entre des formes natives et non-natives, telles que le suremploi du présent dans des contextes où on s'attendrait à un marqueur du passé ${ }^{2}$ :

(1) Quand j'étais à Paris, je reste chez mon ami

Pourtant, bien qu'il s'agisse d'une alternance différente, la variation linguistique constitue également une caractéristique importante chez le locuteur natif. À la différence $\mathrm{du}$ locuteur L2, la variation chez le locuteur natif consiste en le choix entre une variante formelle et une ou plusieurs autres variantes informelles. Faisant confiance à la compétence sociolinguistique qu'il s'est appropriée, le locuteur natif sait faire le bon choix pour produire un discours approprié selon le contexte où il parle. La variation sociolinguistique est évidente dans toutes les composantes de la langue, à savoir la syntaxe (par exemple, le choix entre les différentes formes interrogatives telles que 'estce que' et l'inversion ${ }^{3}$ ), la morphologie (par exemple, l'effacement du 'ne' : 'je ne sais pas' v. 'je sais $\operatorname{pas}^{\prime}$ ), le lexique (par exemple, l'alternance entre un lexique familier, tel que 'mec', et un lexique formel, tel que 'homme'), et la phonologie (par exemple, l'effacement $\mathrm{du} / \mathrm{l} / \mathrm{:}$ ' 'il y a' v. 'y a's).

Grâce à leurs analyses quantitatives, de nombreuses études de la variation chez le locuteur natif indiquent que le choix que fait celui-ci entre les différentes variantes ne se fait pas de façon aléatoire, mais est contraint de façon probabiliste et non catégorique par 
différents facteurs linguistiques, stylistiques, et socio-biographiques ${ }^{6}$. Il s'ensuit que l'acquisition de la variation consiste non seulement en l'appropriation des différentes variables de la langue cible, mais aussi en l'appropriation des différents facteurs qui contraignent l'emploi des variantes dans la langue cible. De ce point de vue, les études antérieures mettent en évidence la difficulté que pose l'acquisition de la variation native au locuteur L27 . Il s'agit principalement des études engagées sur des apprenants adolescents qui suivaient un programme d'immersion en français au Canada, ainsi que du vaste travail de Jean-Marc Dewaele et de Vera Regan sur des apprenants flamands et anglophones du français au niveau universitaire ${ }^{8}$. Ces travaux indiquent tous, de façon générale, que les variantes informelles, tels que l'effacement du 'ne' ou du $/ \mathrm{l} /$, font défaut dans les lectes d'apprenants non-avancés de sorte qu'ils semblent ne disposer que d'un seul registre en français (Dewaele et Regan 2002:124). Même chez les apprenants avancés, on note que leur taux d'emploi est inférieur à celui du locuteur natif de sorte que ceux-ci sur-emploient les variantes formelles même dans des situations où les variantes informelles seraient socialement plus appropriées. Pourtant, bien qu'ils les sousemploient, les locuteurs L2 semblent respecter de façon générale les mêmes facteurs (extra)linguistiques qui contraignent leur emploi dans le discours natif.

Toutefois, même si ces travaux importants sur l'acquisition de la variation engagés par les chercheurs cités depuis dix ans nous permettent de dégager les principaux acquis de la recherche sur l'appropriation de la variation en français L2, il faut des études plus variées sur des apprenants dans différents milieux d'acquisition, et des analyses d'autres variables pour mieux en cerner les caractéristiques éventuellement universelles. Ainsi, l'étude que nous présentons ici a pour but de caractériser l'appropriation de la variation phonétique, jusqu'ici très peu étudiée en français L2, du point de vue de l'emploi de la liaison qui, comme nous le verrons, constitue un phénomène intéressant à étudier en raison de certaines de ses caractéristiques qui la distinguent de la variation morphologique ou syntaxique, par exemple.

\section{La liaison en français}

5 Comme l'indique Bybee (2001:337), la liaison consiste en « the appearance of a word-final consonant before a vowel-initial word in words that in other contexts end in a vowel » [la réalisation de la dernière consonne d'un mot qui est suivi d'un autre mot qui commence par une voyelle, et qui ne se réaliserait pas dans d'autres contextes]. De nombreuses études du locuteur natif telles que Ashby (1981, 2003), Booij and deJong (1987), Bybee (2001), Gadet (1995), and Malécot (1975), parmi d'autres, indiquent qu'il s'agit d'un phénomène complexe de sorte que sa réalisation peut être obligatoire, comme dans l'exemple (2), facultative ou variable, comme dans l'exemple (3), et même défendue selon le contexte, comme dans les exemples (4-8). En ce qui concerne les contextes empêchant l'emploi de la liaison, nous faisons référence aux contextes suivants - devant un $<\mathrm{h}>$ aspiré (4); après la conjonction 'et' (5), après un chiffre (6); entre un substantif et un adjectif au singulier (7); et après un nom propre (8), comme dans les exemples suivants :
(2) Les enfants
(3) Pendant une heure
(4) Les huttes
(5) Une bière et un café
(6) Après huit heures
(7) Un discours ennuyant 
(8) Charles a parlé

Bien que nous n'ayons affaire ici qu'à quelques cas exceptionnels, les études antérieures de la liaison indiquent qu'il s'agit d'un phénomène bien plus complexe. Ces études - dont certaines sont basées sur des analyses quantitatives - ont identifié des cas où la liaison semble être nécessaire, la liaison dite obligatoire, en raison de sa production catégorique par le locuteur natif, tels que dans les exemples suivants - après un article dans une phrase nominale (9); entre un pronom sujet et le verbe qui le suit (10); après des prépositions monosyllabiques (11); et dans des expressions figées (12) :

(9) Les enfants

(10) Vous avez vu

(11) Dans une heure

(12) De temps en temps

7 Pourtant, il est à noter que, bien que ces contextes soient prototypiquement considérés comme des contextes catégoriques, certaines études, à savoir Ashby (1981a, 2003) et Green et Hintze (2001) indiquent que la liaison ne s'y produit pas universellement de façon aussi catégorique qu'on ne l'aurait prévu. Par exemple, pour certains de leurs intervenants, bien qu'il soit fort élevé, le taux d'emploi n'atteint pas $100 \%$ dans les contextes des formes figées et entre un article et un substantif, de sorte que ces contextes dits obligatoires semblent témoigner eux aussi d'une certaine variation.

8 À la différence de ces cas de liaison dite obligatoire où la liaison se produit de façon quasicatégorique, d'autres exemples indiquent qu'il ne s'agit pas du même phénomène, de sorte que la liaison se produit de façon variable. Ainsi, comme dans les exemples suivants de liaison dite variable ou facultative, le locuteur ne produit la liaison que quelquefois par exemple, entre un verbe et son complément (13); entre un sujet nominal et un verbe (14); après des particules négatives (15); et après l'auxiliaire 'être' (16) :

(13) Nous regardons un film

(14) Les enfants avaient fini

(15) Il n'est pas ingénieur

(16) Ils sont arrivés

Des études antérieures telles que celles de Ågren (1979), Armstrong (2001, 2002), Ashby (1981, 2003), Bybee (2001), Booij et deJong (1987), Encrevé (1988), Green et Hintze (1988, 2001), Klausenberger (1984), Morin et Kaye (1982), et Smith (1998) indiquent que dans le cas de la liaison variable, le choix que fait le locuteur natif entre la production de la liaison ou non est contraint par des facteurs multiples, produisant ainsi une alternance très complexe. Par exemple, en ce qui concerne les facteurs linguistiques, Ashby a identifié, dans son étude du français tourangeau, de nombreux facteurs phonétiques, lexicaux, et syntaxiques qui influencent le choix que font les locuteurs natifs. En ce qui concerne les facteurs phonologiques, on note, par exemple, que la liaison ne se produit pas de façon uniforme avec les différents phonèmes de liaison, mais, à l'inverse, certains phonèmes favorisent sa production plutôt que d'autres. Quand il s'agit $d u / n /$, la production de la liaison est plus fréquente que dans le cas $\mathrm{du} / \mathrm{t} / \mathrm{ou} \mathrm{du} / \mathrm{z} /$. Sa production est plutôt rare quand il s'agit des phonèmes $/ \mathrm{k} /, / \mathrm{p} /$, et $/ \mathrm{r} /$. De façon analogue, Ashby trouve que le phonème qui précède le phonème de liaison influence le choix que fait le locuteur de produire la liaison ou non. De ce point de vue, nous notons, par exemple, que la liaison est plus fréquente si elle suit des voyelles orales et nasales, alors que des consonnes défavorisent sa production. Booij et deJong (1987) notent également que des facteurs rythmiques influencent la variation qu'on observe à l'emploi de la liaison par le locuteur natif. Par exemple, quand l'énoncé où peut se produire la liaison termine la 
phrase, sa production semble être plus favorisée que dans les cas où l'énoncé se trouve à l'intérieur de la phrase, comme dans l'exemple suivant que les auteurs nous fournissent :

(17) ils travaillent d'abord et mangent_après

(18) d'habitude, ils mangent_après avoir joué leur partie d'échecs

10 En ce qui concerne l'influence des facteurs lexico-phonétiques, nous notons que la longueur relative des mots dans l'énoncé où peut se produire la liaison influence également sa production ou non. Par exemple, dans les cas où le mot suivant est plus long que le premier mot et quand les mots sont également de la même longueur, le taux d'emploi de la liaison est plus élevé que dans les cas où le premier mot est le plus long.

11 Du point de vue syntaxique, la variation qu'on observe à l'emploi de la liaison a suscité plusieurs discussions dans des articles de recherche. De ce point de vue, l'emploi de la liaison varie de façon importante selon la relation syntaxique entre les mots qui constituent l'énoncé où peut se produire la liaison. Par exemple, on identifie des cas où sa production est plutôt rare, tels que, après une préposition polysyllabique ou après un verbe à l'infinitif. En revanche, d'autres contextes constituent des contextes plus favorables à sa production, bien qu'il ne s'agisse pas d'une production catégorique, comme par exemple, après le verbe 'être'.

Finalement, mis à part ces facteurs linguistiques contraignant le choix fait par le locuteur de la réalisation ou non de la liaison, des facteurs extralinguistiques et stylistiques entrent également en jeu. Par exemple, en tant que marqueur prestigieux, la liaison se produit plus fréquemment pendant un style formel qu'informel. En ce qui concerne les facteurs socio-biographiques, nous notons également que sa production est plus élevée chez les locuteurs des classes moyennes et chez les locuteurs plus âgés de sorte que la liaison témoigne d'un certain déclin chez les locuteurs plus jeunes qui la produisent moins fréquemment aujourd'hui que dans le passé. En ce qui concerne le rôle du sexe du locuteur sur l'emploi de la liaison, le résultat des études témoigne d'une certaine divergence, ce qui en nous permet pas de tirer des conclusions définitives : par exemple, selon Ashby $(1981,2003)$ et Green et Hintze (2001), bien qu'il s'agisse d'un marqueur prestigieux, la production de la liaison ne correspond pas à la tendance générale en sociolinguistique qui veut que les locutrices produisent les marqueurs prestigieux plus fréquemment que les locuteurs. Ces chercheurs notent que son taux d'emploi est plus élevé chez les locuteurs que chez les locutrices. En revanche, Booij et deJong (1987) et Malécot (1975) proposent des résultats qui s'opposent à cette tendance, alors que Smith (1998) ne trouve pas de différences entre les femmes et les hommes.

13 Somme toute, les caractéristiques de l'emploi de la liaison qui veulent en faire un phénomène catégorique aussi bien que variable, contraint par plusieurs facteurs linguistiques, stylistiques et extralinguistiques, indiquent qu'il s'agit d'un phénomène très complexe chez le locuteur natif. Cela s'avère vrai, sans même noter que la tendance $\mathrm{du}$ locuteur natif à suremployer la liaison dans des contextes où son usage est typiquement défendu. Ce phénomène est tout particulièrement apparent dans le cas du / $\mathrm{z} /$ pour marquer le pluriel, comme lors de sa réalisation fautive dans 'une personne/z/ âgée'.

14 En raison de la complexité de l'emploi de la liaison par le locuteur natif, une étude de son appropriation par les locuteurs L2 sert à approfondir nos connaissances de leur acquisition de la variation en L2 par rapport à une variable dont les caractéristiques la distinguent nettement d'autres variables qu'ont étudiées les recherches antérieures. Par exemple, il s'agit dans ce cas d'un phénomène à la fois variable et catégorique. Par 
ailleurs, à la différence d'autres variables qui consiste en l'effacement d'une variante formelle, l'acquisition de la liaison consiste, par contre, en la réalisation d'une variante prestigieuse. Ainsi, du point de vue de l'emploi de la liaison par des locuteurs L2, cet article a pour but de présenter les résultats d'un projet qui vise à caractériser l'acquisition de la variation par des apprenants avancés du français dans différents milieux acquisitionnels. Nous présentons dans la section suivante le projet entrepris.

\section{Présentation de l'étude}

\subsection{Les intervenants}

Pour l'étude que nous présentons ici, nous avons analysé les données orales de 12 intervenants guidés qui participaient à un projet sur l'acquisition du français par des apprenants anglophones en Irlande9. À l'époque où nous avons recueilli ces données, les intervenants étaient des étudiants qui se spécialisaient dans le français à l'université où ils l'avaient appris pendant 2-3 ans, et pendant 5-6 ans au lycée auparavant. Tous nos apprenants hibernophones connaissaient aussi le gaélique puisqu'ils l'avaient tous appris à l'école pendant 12 ans environ. À l'université, leur programme d'études en français consistaient en des cours formels de langue écrite aussi bien qu'en des cours de langue parlée avec des locuteurs natifs, où on se servait de documents visuels et audios authentiques, tels que des articles de journaux et des émissions de télévision.

Bien qu'il s'agisse d'apprenants guidés, certains d'entre eux avaient par ailleurs passé un long séjour en France, où ils avaient suivi un programme d'études d'un an à l'université dans le cadre d'un échange international. Pendant ce séjour, les intervenants n'avaient pas suivi de cours formels pour des étrangers sur la langue française, mais assistaient aux mêmes cours que leurs camarades français. En raison de ces différences concernant leur niveau de contact avec la langue cible selon que les apprenants avaient passé un long séjour en France ou non, nous avons identifié deux groupes d'apprenants : dans le groupe 1 , il s'agit d'apprenants qui avaient suivi deux ans d'enseignement à l'université. Dans le groupe 2, il s'agit d'apprenants qui avaient exactement le même niveau d'enseignement, mais qui avaient, par ailleurs, passé un long séjour en France dans le cadre du programme d'études que nous venons de détailler. En considérant les résultats de chaque groupe, nous pourrons considérer le rôle éventuel du contact informel que permet un tel séjour en France sur l'acquisition de la liaison par les apprenants.

Du point de vue de leur niveau linguistique, il s'agit d'apprenants avancés, au sens de Bartning (1997), puisque la morphologie était déjà émergée dans leurs lectes ${ }^{10}$. C'est-àdire, bien qu'employant de façon variable et distincte du locuteur natif, en général, les apprenants produisent toutes les formes morphologiques et syntaxiques de la langue cible. Par ailleurs, du point de vue de leurs caractéristiques personnelles, les apprenants correspondent à ce profil d'apprenant avancé guidé, défini par Bartning : dans un premier temps, à la différence des apprenants naturels, il s'agit d'apprenants qui ont appris le français pendant une longue période dans un milieu formel à l'étranger, de sorte que leurs connaissances métalinguistiques étaient très développées. Dans un deuxième temps, les apprenants guidés se distinguent des apprenants naturels puisque, ayant choisi de se spécialiser en français pour leur programme d'études, ces apprenants sont, pour des raisons professionnelles, motivés dans leur apprentissage du français. 


\subsection{Le recueil des données}

18 Pour la collecte des données, les intervenants ont participé à un entretien sociolinguistique avec le chercheur anglophone, qui était un locuteur quasi-natif du français. Chaque entretien a duré environ une heure, et a suivi le réseau de modules conversationnels proposés par Labov (1984) dans le cadre de l'enquête sociolinguistique qui vise à recueillir des données naturelles et spontanées. Pourtant, afin de mieux correspondre aux intérêts spécifiques des intervenants, certains des sujets de conversation ont été adaptés : il s'agissait de sujets formels aussi bien qu'informel, tels que les passe-temps, les vacances d'été et des visites à l'étranger, les études à l'université, les projets professionnels et personnels, les relations franco-irlandaises, et le module célèbre de Labov, 'le risque de la mort'. Suite à la collecte des données, elles ont été transcrites en orthographe standard en suivant les modalités de transcription proposées par Blanche-Benveniste et Jeanjean (1986).

\subsection{L'analyse des données et leur codage}

Pour l'étude que nous présentons ici de la variation phonologique caractérisant l'emploi de la liaison par le locuteur L2, l'analyse des données consistait à y répertorier tous les énoncés qui constituaient des contextes où pouvait s'employer la liaison, même si les apprenants ne l'y produisaient pas. Il s'agissait de contextes variables aussi bien qu'invariables, comme dans les exemples 9-16 ci-dessus. Dans le cas des énoncés où il n'était pas clair pour des raisons auditoires si les intervenants produisaient la liaison ou non, ils ont été exclus de l'analyse.

Dans le cadre d'une analyse variationniste qui vise à mettre en lumière les facteurs linguistiques et extralinguistiques qui contraignent la variation linguistique chez le locuteur, tous les énoncés ont été codés pour différents facteurs qui avaient été identifiés auparavant comme des contraintes sur la variation à l'emploi de la liasion par le locuteur natif. Comme nous l'avons vu ci-dessus, il s'agit :

- des facteurs phonétiques telle que l'influence du phonème de liaison sur la réalisation ou non de la liaison;

- des facteurs lexico-phonétiques, comme l'influence de la longueur des mots concernés;

- des facteurs grammaticaux, à savoir les rapports syntaxiques entre les mots concernés;

- des facteurs stylistiques selon qu'il s'agit d'un style formel ou informel;

- et des facteurs extralinguistiques concernant les caractéristiques socio-biographiques des locuteurs tels que l'âge, le sexe, et la classe sociale.

21 Faute d'espace, nous limiterons notre présentation des résultats à l'influence de facteurs grammaticaux sur l'emploi de la liaison par les apprenants. Il s'agit de voir si certains contextes syntaxiques favorisent l'emploi de la liaison alors que d'autres le défavorisent comme chez le locuteur natif. Les exemples de contextes syntaxiques où pouvait s'employer la liaison dite obligatoire dans les données sont fournis ci-dessous :

(19) entre un article et un substantif : les enfants

(20) entre un pronom clitique et un verbe : ils ont parlé

(21) après un adverbe monosyllabique et un adjectif : très intéressant

(22) après une préposition monosyllabique : dans un instant

(23) dans une forme figée : de temps en temps

(24) entre un adjectif et un substantif: un bon enfant 
(25) entre un pronom objet et un verbe : je les ai vus

\section{suivants :}

(26) après quand (conjonction non-interrogative) : quand ils parlaient

(27) après un adverbe polysyllabique : nous nous sommes beaucoup amusés

(28) après une préposition polysyllabique : pendant une heure

(29) après être (verbe non-auxiliaire) : c'est intéressant

(30) entre un substantif et un adjectif : des enfants aimables

(31) après un auxiliaire monosyllabique : ils sont arrivés

(32) après un auxiliaire polysyllabique : vous avez entendu

(33) après une particule négative : ils ne sont pas arrivés

(34) entre un verbe et son complément : nous lisons un livre

(35) entre un substantif et un verbe : les gens entendaient mal

(36) après une conjonction : puis ils sont sortis

Suite au codage des données, l'analyse quantitative des données s'est faite de façon à analyser la variation à l'emploi de la liaison par les apprenants dans ces différents contextes syntaxiques.

\subsection{Résultats}

Le tableau suivant indique le taux d'emploi des liaisons obligatoire et variable par les apprenants dans les groupes 1 (qui n'avaient pas fait de long séjour en France) et 2 (qui avaient passé un an en France).

\begin{tabular}{|l|l|l|}
\hline & Obligatoire & Variable \\
\hline Groupe 1 & $82.2 \%$ & $8.2 \%$ \\
\hline Groupe 2 & $95 \%$ & $12.7 \%$ \\
\hline
\end{tabular}

\section{Tableau 1}

\% Liaisons réalisées dans des contextes obligatoires et variables

Il s'ensuit de ce tableau que le séjour passé en France par les apprenants dans le groupe 2 a été très bénéfique à leur acquisition de la liaison : dans le cas de la liaison obligatoire aussi bien que dans le cas de la liaison variable, nous notons que son taux de réalisation est plus élevé par rapport aux apprenants qui n'étaient pas allés en France. Ces différences sont significatives dans chaque cas $(t=2.452 ; \mathrm{p}<0.05 ; \mathrm{t}=4.7 ; \mathrm{p}<0.05)$. Cela est tout particulièrement le cas en ce qui concerne la liaison obligatoire de sorte que les apprenants dans le groupe 2 la produisent de façon quasi-catégorique (95\%), alors que le taux d'emploi de $82,2 \%$ chez les autres apprenants indique qu'ils produisent la liaison dite catégorique de façon variable. Toutefois, à la différence de la liaison obligatoire, l'emploi de la liaison variable est très peu élevé dans les deux groupes par rapport aux locuteurs natifs, comme ceux de l'étude d'Ashby (2003), du français parlé tourangeau, note un taux d'emploi de $28 \%$.

Pour mieux cerner les caractéristiques de l'emploi de la liaison par les apprenants dans les différents contextes syntaxiques que nous avons identifiés ci-dessus, nous considérons 
dans un premier temps l'emploi de la liaison obligatoire, et dans un deuxième temps, l'emploi de la liaison variable. Ceci nous permettra de voir si les apprenants produisent la liaison de façon uniforme dans tous les contextes ou si certains contextes favorisent sa production plus que d'autres.

\subsection{La liaison obligatoire}

Le tableau suivant présente le taux d'emploi de la liaison dite obligatoire dans ses différents contextes d'emploi pour chaque intervenant dans notre étude.

\begin{tabular}{|l|l|l|l|l|l|l|}
\hline Groupe 1 & & & & & & \\
\hline Apprenant & 1 & 2 & 3 & 4 & 5 & 6 \\
\hline Article + substantif & $96 \%$ & $70 \%$ & $70 \%$ & $94 \%$ & $95 \%$ & $97 \%$ \\
\hline Pronom sujet + verbe & $100 \%$ & $93 \%$ & $100 \%$ & $92 \%$ & $100 \%$ & $80 \%$ \\
\hline Préposition monosyllabique & $100 \%$ & $82 \%$ & $73 \%$ & $78 \%$ & $96 \%$ & $75 \%$ \\
\hline Adverbe monosyllabique & $22 \%$ & $25 \%$ & $0 \%$ & $100 \%$ & $100 \%$ & $33 \%$ \\
\hline forme figée & $50 \%$ & $40 \%$ & $44 \%$ & $50 \%$ & $61 \%$ & $50 \%$ \\
\hline Adjectif + substantif & $50 \%$ & $0 \%$ & $0 \%$ & - & $0 \%$ & $0 \%$ \\
\hline Pronom d'objet & $50 \%$ & $0 \%$ & 0 & $0 \%$ & $0 \%$ & $0 \%$ \\
\hline
\end{tabular}

\begin{tabular}{|l|l|l|l|l|l|l|}
\hline Groupe 2 & & & & & & \\
\hline Apprenant & 1 & 2 & 3 & 4 & 5 & 6 \\
\hline Article + substantif & $93 \%$ & $100 \%$ & $96 \%$ & $100 \%$ & $95 \%$ & $97 \%$ \\
\hline Pronom sujet + verbe & $100 \%$ & $100 \%$ & $99 \%$ & $97 \%$ & $97 \%$ & $100 \%$ \\
\hline Préposition monosyllabique & $100 \%$ & $100 \%$ & $94 \%$ & $92 \%$ & $100 \%$ & $100 \%$ \\
\hline Adverbe monosyllabique & $20 \%$ & $66 \%$ & $0 \%$ & $50 \%$ & $60 \%$ & $33 \%$ \\
\hline Forme figée & $100 \%$ & $100 \%$ & $100 \%$ & $100 \%$ & $85 \%$ & $100 \%$ \\
\hline Adjectif + substantif & - & $33 \%$ & $20 \%$ & $29 \%$ & $13 \%$ & $0 \%$ \\
\hline Pronom d'objet & - & $33 \%$ & $20 \%$ & $29 \%$ & $13 \%$ & $0 \%$ \\
\hline
\end{tabular}

Tableau 2

\% Liaisons obligatoires réalisées selon le contexte syntactique 
28

Ces résultats nous permettent de dégager quelques tendances intéressantes concernant l'acquisition de la liaison : nous notons tout particulièrement que l'emploi de la liaison obligatoire n'est pas du tout un phénomène uniforme chez les apprenants, mais, au contraire, varie de façon significative selon le contexte syntaxique. Par exemple, le taux d'emploi de la liaison par tous les apprenants est très peu élevé entre un adjectif et un substantif, et après un pronom objet, bien que les apprenants dans le groupe 2 la produisent plus fréquemment que les autres apprenants. Voire, dans le groupe 1, la liaison fait plus ou moins défaut dans ces contextes, aussi bien que dans le cas des expressions figées. En ne produisant pas la liaison dans ces contextes, les apprenants se distinguent énormément du locuteur natif, chez qui il s'agit de contextes obligatoires, nécessitant l'emploi de la liaison. Toutefois, les autres contextes, c'est-à-dire, entre un article et un substantif, entre un pronom sujet et un verbe, et après une préposition monosyllabique, posent moins de difficulté pour la production de la liaison. Cela est tout particulièrement vrai pour les apprenants du groupe 2 qui ont atteint un taux d'emploi de plus de $90 \%$ dans chaque contexte, ce qui n'est pas forcément le cas chez les apprenants de l'autre groupe qui ont encore du progrès à faire. Finalement, nous notons que les apprenants se distinguent beaucoup en ce qui concerne leur emploi de la liaison après des adverbes monosyllabiques de sorte que dans ce contexte certains produisent la liaison bien plus fréquemment que d'autres.

\subsection{La liaison variable}

Les taux d'emploi de la liaison dite facultative dans ses différents contextes d'emploi sont présentés dans le tableau suivant.

\begin{tabular}{|l|l|l|l|l|l|l|}
\hline Groupe 1 & & & & & & \\
\hline Apprenant & 1 & 2 & 3 & 4 & 5 & 6 \\
\hline 'quand' & - & $0 \%$ & - & $0 \%$ & $0 \%$ & $0 \%$ \\
\hline Adverbe polysyllabique & - & - & - & $0 \%$ & $0 \%$ & $0 \%$ \\
\hline Préposition polysyllabique & - & - & $0 \%$ & $0 \%$ & $100 \%$ & $0 \%$ \\
\hline 'être' & $4 \%$ & $0 \%$ & $0 \%$ & $11 \%$ & $21 \%$ & $0 \%$ \\
\hline Substantif + adjectif & $33 \%$ & $66 \%$ & - & $0 \%$ & $0 \%$ & $0 \%$ \\
\hline Auxiliaire monosyllabique & $50 \%$ & $0 \%$ & $60 \%$ & $0 \%$ & $0 \%$ & $0 \%$ \\
\hline Particule négative & $0 \%$ & $0 \%$ & $0 \%$ & $100 \%$ & $16 \%$ & $0 \%$ \\
\hline Verbe + complément & $0 \%$ & $0 \%$ & $0 \%$ & $0 \%$ & $0 \%$ & $3 \%$ \\
\hline Auxiliaire polysyllabique & - & $0 \%$ & - & $0 \%$ & $0 \%$ & $0 \%$ \\
\hline Substantif + verbe & - & $0 \%$ & $0 \%$ & $0 \%$ & $0 \%$ & $0 \%$ \\
\hline
\end{tabular}

Corela, HS-1 | 2005 


\begin{tabular}{|l|l|l|l|l|l|l|}
\hline Conjonction & $18 \%$ & $8 \%$ & $0 \%$ & $17 \%$ & $0 \%$ & $0 \%$ \\
\hline
\end{tabular}

\begin{tabular}{|l|l|l|l|l|l|l|}
\hline Groupe 2 & & & & & & \\
\hline Apprenant & 1 & 2 & 3 & 4 & 5 & 6 \\
\hline \hline 'quand' & $0 \%$ & $0 \%$ & $0 \%$ & $0 \%$ & $17 \%$ & $0 \%$ \\
\hline Adverbe polysyllabique & $0 \%$ & $0 \%$ & $0 \%$ & $0 \%$ & $0 \%$ & - \\
\hline \hline Préposition polysyllabique & $0 \%$ & $25 \%$ & - & - & $0 \%$ & - \\
\hline 'être' & $24 \%$ & $10 \%$ & $2 \%$ & $11 \%$ & $12 \%$ & $15 \%$ \\
\hline Substantif + adjectif & $0 \%$ & $14 \%$ & $0 \%$ & $20 \%$ & - & - \\
\hline \hline Auxiliaire monosyllabique & $76 \%$ & $46 \%$ & $81 \%$ & $100 \%$ & $31 \%$ & $54 \%$ \\
\hline Particule négative & $18 \%$ & $17 \%$ & $28 \%$ & $20 \%$ & $13 \%$ & $7 \%$ \\
\hline \hline Vuxiliaire polysyllabique & $0 \%$ & $0 \%$ & - & - & - & $0 \%$ \\
\hline Conjonction & $4 \%$ & $5 \%$ & $0 \%$ & $5 \%$ & $2 \%$ & $2 \%$ \\
\hline
\end{tabular}

Tableau 3

\% Liaisons variables réalisées selon le contexte syntactique

Comme dans le cas de la liaison obligatoire, la production de la liaison varie énormément selon le contexte syntaxique. La tendance principale qui se dégage de ce tableau concerne l'absence complète de liaison dans certains contextes chez les apprenants du groupe 1 aussi bien que du groupe 2. Il s'agit tout particulièrement des contextes suivants: après 'quand'; après des adverbes et des prépositions polysyllabiques; entre un verbe et son complément; après des auxiliaires polysyllabiques; et entre un substantif et un verbe. Dans les autres contextes, à savoir après des auxiliaires monosyllabiques, des particules négatives, et des conjonctions, nous notons que, bien que tous les apprenants produisent la liaison, les apprenants du groupe 2 témoignent d'un taux d'emploi plus élevé que les apprenants du groupe 1 . Ainsi, ces contextes s'avèrent plus favorables à l'emploi de la liaison de sorte qu'ils posent moins de difficulté aux apprenants. Cela vaut également pour le verbe 'être'. Finalement, en ce qui concerne le contexte de l'emploi de la liaison entre un substantif et un adjectif, nous notons des différences importantes entre les apprenants dont certains produisent la liaison, alors que d'autres ne la produisent pas. 


\section{Discussion et conclusions}

31 En général, en ce qui concerne la liaison obligatoire aussi bien que la liaison facultative, les résultats indiquent des différences importantes entre les apprenants et le locuteur natif. Bien qu'ils réalisent, en général, la liaison obligatoire de façon quasi-catégorique comme le locuteur natif, cela n'est pas le cas dans tous les contextes obligatoires, dont certains posent une difficulté importante aux apprenants, à savoir dans le cas des expressions figées, entre un substantif et un adjectif, et après les pronoms objets directs. Du point de vue des expressions figées, il est intéressant de noter que le taux peu élevé de l'emploi de la liaison avec elles s'oppose à la tendance générale en acquisition de la variation sociolinguistique qui veut qu'elles constituent un contexte très favorable, même précoce, pour l'emploi des variantes sociolinguistiques. Par exemple, dans son étude de l'effacement du 'ne', Regan (1997) note que ses intervenants irlandais suremploient tout particulièrement les expressions figées, telles que 'je sais pas' et 'il y a pas', pour témoigner d'un taux d'effacement beaucoup plus élevé par rapport à d'autres contextes où l'effacement pourrait se faire ${ }^{11}$.

Les apprenants témoignent d'une difficulté analogue en ce qui concerne la liaison facultative, dont certains contextes dans leur production en font nettement défaut, bien qu'il s'agisse de contextes où son taux d'emploi est plus ou moins élevé chez le locuteur natif. Ainsi, dans son étude du locuteur natif, Ashby (2003) trouve que les taux d'emploi de la liaison varient énormément d'un contexte à l'autre, s'élèvant jusqu'à $85 \%$ après 'quand', mais tombant à $2 \%$ entre un verbe et son complément. Entre ces deux extrêmes, ses résultats indiquent, par exemple, un taux d'emploi de $33 \%$ après des formes de 'être', et un taux d'emploi de $5 \%$ après les prépositions et les adverbes polysyllabiques, aussi bien qu'entre un substantif et l'adjectif qui le suit. Ces différences qui font que l'apprenant se distingue nettement du locuteur natif constituent des zones de fragilité (Bartning, 1997) importantes dans l'acquisition de la liaison par l'apprenant L2. Voire, bien que nos apprenants ayant fait un long séjour en France témoignent d'un taux de réalisation de la liaison plus élevé que les autres intervenants dans l'étude, ils ne se rapprochent tout d'abord pas du locuteur natif du point de vue de leur taux d'emploi général de la liaison. Dans un deuxième temps, pourtant, ils s'éloignent nettement du locuteur natif en raison de l'absence totale de liaison dans certains contextes syntaxiques. Ceci a pour conséquence d'engendrer un sentiment de non respect de toutes les contraintes sur l'emploi de la liaison chez le locuteur natif.

De ce point de vue, nos résultats se distinguent de façon générale de ceux des études antérieures sur l'acquisition de la variation sociolinguistique en langue seconde. Par exemple, dans leur survol des études précédentes, Mougeon et coll. (2003: 27) tirent la conclusion générale que « d'une façon générale, les apprenants FL2 avancés respectent les contraintes linguistiques de la variation » qui sont partagées par l'ensemble des locuteurs au sein de la communauté linguistique. De toute évidence, cela n'est pas le cas par rapport à l'acquisition de la liaison, de sorte que nos apprenants semblent ignorer l'emploi de la liaison facultative dans un large éventail de contextes syntaxiques ${ }^{12}$. Par ailleurs, une autre conclusion tirée par Mougeon et coll. indiquant que « les apprenants avancés FL2 sur-utilisent les variantes standard marquées» (p. 23) ne semble pas valoir dans le cas de l'acquisition de la liaison par nos apprenants. En sous-employant la liaison très nettement par rapport au locuteur natif, il s'agit, au contraire, d'un sur-emploi 
important de la variante non-standard, ce qui s'oppose à la tendance générale en acquisition de la variation sociolinguistique.

Pour comprendre pourquoi ces conclusions s'opposent aux résultats de cette étude, il faut se rendre compte de la spécificité de la variante qu'est la liaison. À la différence d'autres variantes dont l'acquisition consiste en l'effacement de la variante standard, comme par exemple du 'ne' ou du /l/ pour réaliser la variante non-standard, l'acquisition de la liaison consiste, en revanche, en la production de la variante standard qui s'oppose à la variante non-standard, c'est-à-dire l'absence de la liaison qui fait partie au départ de l'interlangue de l'apprenant. Autrement dit, il s'agit du processus inverse, faisant que l'apprenant acquiert la variante non-standard avant que la variante standard ne se manifeste. Cette spécificité du processus d'acquisition pourrait expliquer en grande partie les raisons pour lesquelles les apprenants qui avaient passé un long séjour en France témoignent de très peu de progrès, ce qui fait qu'une dernière conclusion que tirent Mougeon et coll. ne leur correspond pas. Ces auteurs indiquent que «l'exposition au français en dehors de l'école par le biais d'interactions avec les locuteurs natifs favorise l'apprentissage des variantes vernaculaires et des variantes non-standard marquées » (p. 30), de sorte que, dans le cas de notre étude, on peut s'attendre à ce que nos apprenants 'dans le bain' progressent plus par rapport à leur acquisition de la liaison qu'en réalité.

Pourtant, il y a des raisons fortes pour que nos apprenants s'empêchent, d'un côté, d'acquérir la liaison, c'est-à-dire la variante standard, et, ce faisant, pour qu'ils tendent à produire la variante non-standard, c'est-à-dire ne pas produire la liaison. Comme l'indiquent Dewaele et Regan (2002), l'interaction en milieu naturel facilite l'acquisition des variantes non-standard, en raison de ce que leur acquisition constitue un moyen de " faire natif », en s'appropriant " une valeur symbolique d'intégration dans la communauté native » (Regan, 1997 : 206). Ainsi, d'une certaine façon, l'acquisition de la liaison pourrait être défavorisée en raison de ce que l'emploi de cette variante standard pourrait constituer un moyen de ne pas 'faire natif' en parlant de façon trop formelle. Autrement dit, en suremployant la variante non-standard, c'est-à-dire en ne produisant la liaison que très peu, les apprenants font preuve de la même tendance identifiée pour l'acquisition d'autres variantes non-standard, tel que l'effacement du 'ne' ou du /l/. Voire, en ne manifestant un taux d'emploi général de la liaison variable que de 12,7\%, impliquant également que son taux de non-emploi s'élève à $87,7 \%$, nos apprenants en milieu francophone témoignent d'un sur-emploi important de la variante non-standard par rapport au locuteur natif dont le taux ne s'élève qu'à $72 \%$. Il est à noter que cette interprétation de nos résultats concernant l'acquisition de la liaison facultative constitue également un moyen valable d'interpréter l'emploi plus fréquent de la liaison obligatoire dont font preuve les apprenants en milieu naturel par rapport aux apprenants guidés. Puisqu'il s'agit de la liaison obligatoire que produit de façon catégorique le locuteur natif, sa production par l'apprenant en milieu naturel atteint un niveau quasi-catégorique afin que celui puisse également 'faire natif'. S'il n'en augmentait pas son taux d'emploi, il se rapprocherait nettement du locuteur natif.

En conclusion, bien que notre interprétation des résultats s'accorde avec les conclusions générales des études antérieures, en mettant en lumière l'importance du milieu naturel pour l'acquisition des variantes non-standard, elle souligne également la complexité qui caractérise l'acquisition de la variation sociolinguistique en indiquant, d'un côté, les limites de ce milieu pour l'acquisition de certaines variantes standard, telle la liaison 
facultative, mais d'un autre côté, les avantages d'un séjour en milieu naturel pour l'acquisition par l'apprenant guidé d'autres marqueurs standard, telle que la liaison obligatoire. Comme l'indiquent Coleman (1997) et Freed (1998), de telles évidences de la maîtrise linguistique, complètes ou incomplètes, pendant son séjour en milieu naturel entament des conséquences importantes pour la ré-intégration de l'apprenant en milieu guidé, après son séjour en mileu naturel, pour que son programme d'enseignement corresponde du mieux possible à ses besoins acquisitionnels ${ }^{13}$.

\section{BIBLIOGRAPHIE}

ARMSTRONG, N. (2001). Social and Stylistic Variation in Spoken French: a Comparative Approach. Amsterdam et Philadelphie : Benjamins

ARMSTRONG, N. (2002). «The Influence of Spelling Pronunciation in Two Areas of Oil French: An Indication of Social Convergence ». HINTZE, M-A., T POOLEY et JUDGE, A. (dirs.), French Accents: Phonological and Sociolinguistic Perspectives Londres: AFLS / CILT. 45-72

ASHBY, W. (1981). «French Liaison as a Sociolinguistic Phenomenon ». CRESSEY, W. et NAPOLI, D. (dirs.), Linguistics Symposium on Romance Languages. Washington, DC : Georgetown University Press. 46-57

ASHBY, W. (2003). « La liaison variable en français parlé tourangeau : une analyse en temps réel » . Document présenté au Congrès Annuel de l'AFLS (Association for French Language Studies), Tours, septembre 2003

BARTNING, I. (1997). « l'apprenant dit avancé et son acquisition d'une langue étrangère : tour d'horizon et esquisse d'une caractérisation de la variété avancée ». BARTNING, I. (dir). Acquisition et Interaction en Langue Étrangère. Numéro Spécial : Les apprenants avancés 9, 9-50 BLANCHE-BENVENISTE, C. et JEANJEAN, C. (1986). Le Français parlé : transcription et édition. Paris : Didier Erudition

BLONDEAU, H. et NAGY, N. (1998). « Double marquage du sujet dans le français parlé par les jeunes anglo-montréalais ", JENSEN, J. et VAN HERK, G. (dirs). Actes du Congrès Annuel de l'Association Canadienne de Linguistique Ottawa : Cahiers Linguistiques d'Ottawa. 59-70 BOOIJ, G. et DE JONG, D. (1987). « The Domain of Liaison : Theories and Data ». Linguistics 25, $1005-25$

BYBEE, J. (2001). « Frequency Effects on French Liaison ». BYBEE, J. et HOPPER, P. (dirs). Frequency Effects and the Emergence of Linguistic Structures Amsterdam et Philadelphie : Benjamins. 337-59

COLEMAN, J. (1997). « Residence Abroad within Language Study ». Language Teaching 30, 1-20

COVENEY, A. (1998). « Awareness of Linguistic Constraints on Variable ne Deletion ». Journal of French Language Studies 8, 189-208

DEWAELE, J-M. (1992). « L'omission du ne dans deux styles d'interlangue française » Journal of Applied Linguistics 7,1. 3-17 
DEWAELE, J-M. (1999). « Word Order Variation in Interrogative Structures of Native and NonNative French ». ITL Review of Applied Linguistics 123/4, 161-80

DEWAELE, J-M. (2002). « Using Socio-Stylistic Variants in Advanced French Interlanguage: The Case of nous / on ». Eurosla Yearbook III, 205-26

DEWAELE, J-M. (2003). « Variation, chaos et système en interlangue française ». DEWAELE, J-M. et MOUGEON, R. (dirs). Acquisition et Interaction en Langue Étrangère Numéro spécial : L'Appropriation de la variation par les apprenants du français langue seconde

DEWAELE, J-M et REGAN, V. (2001). « The Use of Colloquial Words in Advanced French Interlanguage ». Eurosla Yearbook I, 51-68.

DEWAELE, J-M. et REGAN, V. (2002). « Maîtriser la norme sociolinguistique en interlangue française : le cas de l'omission variable de 'ne' ». Journal of French Language Studies 12, 123-48.

ENCREVÉ, P. (1988). La Liaison avec et sans enchaînement. phonologie tridimensionelles et usages du français. Seuil.

FREED, B. (1998). « An Overview of Issues and Research in Language Learning in a Study Abroad Setting ». FREED, B. (dir). Frontiers. The Study Abroad Journal. Numéro Spécial : Language Learning in a Study Abroad Context, Fall 1998, 31-60.

GADET, F. (1995). Le Français ordinaire. Armand Colin.

GREEN, J. et HINTZE, M-A. (1988). « A Reconsideration of Liaison and Enchaînement ». University of Essex Occasional Papers in Languages and Linguistics 136-68.

GREEN, J. et HINTZE, M-A. (2002). « The Maintenance of Liaison in a Family Network». HINTZE, MA., T POOLEY et JUDGE, A. (dirs.), French Accents: Phonological and Sociolinguistic Perspectives Londres: AFLS / CILT. 24-44.

HOWARD, M. (1998). « Quasi-Bilingual? The Advanced Learner in Second Language Acquisition research ». Pages 5, 63-77.

HOWARD, M. (1999). « The Advanced Learner: Poor Relation of the Second Language Acquisition Family?». Cahiers AFLS (Association for French Language Studies) 5, 1. 7-26.

HOWARD, M. (2001). « The Effects of Study Abroad on the L2 Learner's Structural Skills: Evidence from Advanced Learners of French ». Eurosla Yearbook I, 123-41.

HOWARD, M. (2001-2). «The Effects of a 'Study Abroad' Programme on the Development of Verb Morphology for the Expression of Past time by the Advanced Learner: A Lexical Approach». Teanga: The Irish Yearbook of Applied Linguistics 20, 29-52.

HOWARD, M. (2002a). « Prototypical and Non-Prototypical Marking in the Advanced Learner's Aspectuo-Temporal System ». Eurosla Yearbook II, 87-113.

HOWARD, M. (2002b). «L'Acquisition des temps du passé en français par l'apprenant dit avancé : une approche lexicale ». LABEAU, E. et LARRIVÉE, P. (dirs). Les Temps du passé français et leur enseignement. Amsterdam et Atlanta : Rodopi [Cahiers Chronos 9]. 181-204.

HOWARD, M. (2002c). « Les interrelations entre les facteurs contextuels contraignant l'emploi variable des temps du passé. une étude d'apprenants avancés du français ». Revue Française de Linguistique Appliquée. Acquisition des Langues : Nouvelles Orientations VII,1. 31-42.

HOWARD, M., LEMÉE, I. et REGAN, V. (2004). «The Acquisition of Phonological Variation in Advanced French Interlanguage: The Case of /1/ Deletion ». Document présenté à l'atelier : 'L'Acquisition de la Compétence Sociolinguistique par l'Apprenant Avancé', Sociolinguistic Symposium 15, Newcastle (Royaume-uni), avril 2004. 
KLAUSENBERG, J. (1984). French Liaison and Linguistic Theory. Stuttgart : Franz Steiner Verlag

LABOV, W. (1984). «Field Methods of the Project on Language Change and Variation ». BAUGH, J. et SCHERZER, J. (dirs). Language in Use: Readings in Sociolinguistics. Englewood Cliffs, NJ:

Prentice Hall. 28-53

MALÉCOT, A. (1975). « French Liaison as a Function of Grammatical, Phonetic, and Paralinguistic Variables ». Phonetica 32, 161-79

MORIN, Y-C. et KAYE, J. (1982). « The Syntactic Bases for French Liaison ». Journal of Linguistics 18, 291-330

MOUGEON, R., NADASDI, T. et REHNER, K. (2003). « État de la recherche sur l'appropriation de la variation par les apprenants avancés du fl2 ou fle ». MOUGEON, R. et DEWAELE, J-M. (dirs).

Acquisition et interaction en langue étrangère Numéro spécial : L'Appropriation de la variation par les apprenants du français langue seconde 7-50

MYLES, F., MITCHELL, R. et HOOPER , J. (1999). « Interrogative Chunks in French L2: A Basis for Creative Construction? ».Studies in Second Language Acquisition 21,1. 39-80

NAGY, N., MOISSET, C. et SANKOFF, G. (1996). « On the Acquisition of Variable Phonology in L2 ». Penn Working Papers in Linguistics 3,1. 111-126

REGAN, V. (1997). « Les apprenants avancés, la lexicalisation, et l'acquisition de la compétence sociolinguistique : une approche variationniste ». BARTNING, I. (dir).Acquisition et interaction en langue étrangère (AILE). Numéro Spécial : Les Apprenants Avancés 9, 193-210

REHNER, K. et MOUGEON, R. (1999). « Variation in the Spoken French of Immersion Students: To ne or Not to ne, That is the Sociolinguistic Question ». Canadian Modern Language Review 56, 124-54

REHNER, K., MOUGEON, R. et NADASDI, T. (2002). « The Learning of Sociolinguistic Variation by Advanced FSL Learners. The Case of 'nous' Versus 'on' in Immersion French ». Studies in Second Language Acquisition 25, 127-56

SMITH, A. (1998). « French Liaison: A Proposed Simplification ». Francophonie 17, 11-14

THOMAS, A. (1998). « La liaison et son enseignement : des modèles orthopiques à la réalité linguistique ». Canadian Modern Language Review 54, 543-52

\section{NOTES}

1. Je tiens à remercier Isabelle Lemée pour ses commentaires précieux sur ce travail.

2. Pour un survol des résultats émanant d'un grand projet sur ce type de variation linguistique, voir Howard (2001, 2001-2, 2002a, 2002b, 2002c).

3. Voir Dewaele (1999).

4. Voir, par exemple, Dewaele et Regan (2002) et Rehner et Mougeon (1999).

5. Voir Howard et coll. (2004).

6. Pour un survol des études en français qui mettent en lumière dans un cadre variationniste le système de facteurs contraignant la variation chez le locuteur natif, voir Armstrong (2001).

7. Pour un survol de cette recherche, voir Mougeon et coll. (2003) et Rehner et coll. (2003).

8. Pour un survol du vaste travail sur les apprenants en immersion au Canada, voir Mougeon et coll. (2003) et Rehner et coll. (2003). Voir également Nagy et coll. (1996). Pour des études spécifiques sur les apprenants anglophones et néerlandophones, voir, parmi d'autres, Dewaele (1992, 2002, 2003), Dewaele et Regan (2001, 2002). 
9. Pour des détails supplémentaires sur le projet, voir Howard (2002b).

10. Pour une discussion supplémentaire du lecte avancé en acquisition des langues secondes, voir Howard $(1998,1999)$.

11. Pour une discussion du rôle important que jouent les expressions figées en acquisition des L2, voir également Myles et coll. (1999).

12. Dans une étude fort innovatrice, Coveney (1998) trouve que les apprenants non seulement respectent les contraintes linguistiques sur la variation, mais en ont des « intuitions ».

13. Voir Thomas (1998) pour une discussion de l'enseignement pédagogique de la liaison.

\section{RÉSUMÉS}

Dans cet article, nous présentons une analyse quantitative de l'emploi variable de la liaison dans différents contextes syntaxiques par des apprenant anglophones de français L2. Les résultats indiquent des différences très nettes entre nos apprenants avancés et le locuteur natif : d'un côté, bien qu'ils produisent la liaison dite obligatoire de façon quasi-catégorique, cela n'est pas le cas dans tous les contextes obligatoires. D'un autre côté, nous notons non seulement que le taux d'emploi de la liaison dite variable est nettement inférieur à celui du locuteur natif, mais son emploi fait défaut dans certains contextes variables. Les résultats sont discutés dans le cadre des principaux acquis qui se dégagent actuellement de la recherche sur l'acquisition de la variation sociolinguistique.

This paper presents a quantitative analysis of the L2 speaker's use of so-called obligatory and variable liaison in different syntactic contexts. Results suggest that our advanced anglophone informants differ considerably from the native speaker: on the one hand, whilst the learner approaches almost categorical use of obligatory liaison, this does not hold true across all obligatory contexts. On the other hand, variable liaison poses considerable difficulty, such that use of the liaison is absent in a number of variable contexts, and even in those contexts where it is produced, its level of usage is considerably inferior to that of the native speaker. The results are discussed in relation to the principal trends identified in recent research on the acquisition of sociolinguistic variation.

\section{AUTEUR}

\section{MARTIN HOWARD}

University College Cork 